\title{
LETTERS
}

doi:10.1017/\$1041610214001525

\section{The use of the Addenbrooke's Cognitive Examination in a community memory clinic}

Early diagnosis of dementia enables early intervention and gives people the opportunity to make choices and plan for their future. Such an early diagnosis requires accurate assessment. A clinical assessment in people with suspected dementia in a memory clinic setting should include a clinical interview of the patient and their next of kin, a physical and neurological examination, cognitive assessment with appropriate cognitive tests, blood investigations, ECG (electrocardiography), and brain imaging.

It can be difficult to find a cognitive test which can identify cognitive impairment as well as provide information on the most likely aetiology. This is because most screening tests have been validated in particular subtypes of dementia. At a later stage their sensitivity and specificity statistics were calculated against normal controls rather than against different causes of dementia (Cullen et al., 2007).

Our aim is to assess the usefulness of the ACE-R (Addenbrooke's Cognitive Examination - revised) in a community memory clinic.

The Addenbrooke's Cognitive Examination (ACE; Mathuranath et al., 2000) was developed to provide a brief test sensitive to the early stages of dementia and, at a later stage, its revised version was validated with different diagnoses (Mioshi et al., 2006). Mioshi et al. (2006) found that a cut-off score of 82 is 100 times more likely to come from a patient with dementia than one without. There is a lack of studies in the literature with larger samples reporting the use of the ACE- $R$ in a community memory clinic.

Two-hundred and six consecutive patients attending a community memory clinic based in Cynon Valley Hospital, South Wales between 2010 and 2012 were examined retrospectively for three parameters: clinical diagnosis, ACE-R results, and radiological brain scan findings. The radiological scanning included brain CT (computed tomography), MRI (magnetic resonance imaging), and SPECT (single photon emission computed tomography) scanning. For the diagnosis of mild cognitive impairment (MCI), Alzheimer's disease (AD), neurovascular dementia (VaD), frontotemporal dementia (FTD), and Lewy bodies dementia (DLB), international criteria were used (McKhann et al., 1984; Roman et al, 1993; Petersen et al., 1997; Neary et al., 1988; McKeith et al., 2005). Our community memory team consists of a consultant psychiatrist, a middle grade doctor, a second-year trainee doctor, a clinical psychologist, and a clinical nurse specialist. We also can refer to other members of the community mental health team including occupational therapists, community psychiatric nurses, and social workers. Feedback and discussion of the final clinical diagnosis and management plan were given to patients and their next of kin as soon as all clinical and investigation results became available. The radiological findings were divided as the following: normal, showing vascular brain insult, showing temporal lobe atrophy, or mixed pathology. The ACE-R results were categorized as normal, $\mathrm{MCI}$, suspect $\mathrm{AD}$, suspect $\mathrm{VaD}$, or suspect FTD (see Table S1, available as supplementary material attached to the electronic version of this paper at www.journals. cambridge.org/jid_IPG). We have tested the ACE$\mathrm{R}$ diagnostic accuracy against the clinical diagnostic standard criteria by calculating the diagnostic odds ratio (OR), 95\% confidence interval, sensitivity, specificity, positive predictive value (PPV), negative predictive value (NPV), and likelihood ratio $(\mathrm{LR}+)$. All $p$-values were calculated using the two-tailed Fisher Exact test.

The average age of the sample was 75.3 years ( $\mathrm{SD}=9.2$; range $=45-99$ years). The mean ACE$\mathrm{R}$ score was 66 out of $100(\mathrm{SD}=15.90$; range $=23$ 96) and the mean years in education were 9.7 years $(\mathrm{SD}=1.63)$. The most common diagnoses were $\mathrm{AD}(37 \%)$, vascular dementia (22\%), and mixed dementia (14\%) with an average duration of cognitive impairment from onset of reported symptoms of 28 months $(S D=15.1)$. We observed that the older the patient the more likely they were to suffer dementia; however, the clinical diagnosis did not differ by age, $F(1,163)=0.725, p=0.396$. More than half $(55 \%)$ of the patients were female $(N=113)$. The clinical diagnosis did not differ by gender, $X^{2}$ $(1, N=206)=1.942, p=0.163$ or by social class, $F(1,163)=0.707, p=0.402$ or years in education, $F(1,163)=0.007, p=0.935$. Pearson correlation showed that the clinical diagnosis correlated with the ACE-R, $r(204)=0.621, p=0.000$; and with radiological findings, $r(195)=0.585, p=0.000$, i.e. there is a close relationship between the results and the clinical diagnosis. Multiple regression analysis showed a significant association between the clinical diagnosis and the ACE-R, $\beta=0.621$, 
$t(202)=11.246, p=0.000$. The clinical diagnosis also explained a significant proportion of the variance among the participants, $R^{2}=$ $0.385, F(1,202)=126.477, p=0.000$. Analysis of covariance to look at the clinical diagnosis adjusted for age, sex, years in education, and social class showed a significant association with the ACE$\mathrm{R}, F(6,163)=12.180, p=0.000$; and with the radiological findings, $F(5,163)=8.728, p=0.000$. When considering the diagnosis as "yes" or "no," the clinical diagnosis was significantly associated with the ACE-R, $X^{2}(6, N=205)=203.534$, $p=0.000$; and with the radiological findings, $X^{2}$ $(5, N=205)=203.367, p=0.000$.

With a cut-off score for diagnosing dementia of 82 , the overall accuracy of the ACE-R was $61 \%$, sensitivity of $80 \%$, specificity of $71 \%$, PPV of $56 \%$, and NPV of $51 \%$. These results differ from the original work of Mathuranath et al. (2000). The authors reported that at a cut-off score of 83, they had an optimal sensitivity (82\%) and specificity (96\%), and maintained a reasonably high PPV and NPV at different prevalence rates of dementia. However, the original paper was administered to a smaller, younger group of patients whereas this study reflects clinical practice in that there was no selection of patients by disease category, no application of exclusion criteria, and the fact that all patients had at least a complaint of impaired cognition (usually of memory) hence their referral. Larner (2005) similarly found that the ACE-R is an easy-to-use and acceptable test for patients, which also has excellent sensitivity. There is scope for further research to evaluate the ACE-R diagnostic accuracy in mixed dementia or DLB.

The authors confirm that the ACE-R is a useful test to use in a community memory clinic as it is practical and simple to use. We believe that there is good evidence for using the ACE-R and currently ACE-III - with regards to early diagnosis. It is the authors' opinion that clinical history, carer interview, neurocognitive assessment, blood investigations, brain scanning, and whenever applicable, biomarkers testing, should be the basic practice in every memory clinic. Clinical judgment is important but when incorporated with all the above it will increase the overall diagnostic accuracy.

\section{Conflict of interest}

None.

\section{References}

Cullen, B., O’Neill, B., Evans, J. J., Coen, R. F. and Lawlor, B. A. (2007). A review of screening tests for cognitive impairment. Fournal of Neurology, Neurosurgery and Psychiatry, 78, 790-799.

Larner, A. J. (2005). An audit of the Addenbrooke's Cognitive Examination (ACE) in clinical practice. International fournal of Geriatric Psychiatry, 20, 593-594.

Mathuranath, P. S., Nestor, P. J., Berrios, G. E., Rakowicz, W. and Hodges, J. R. (2000). A brief cognitive test battery to differentiate Alzheimer's disease and frontotemporal dementia. Neurology, 55, 1613-1620.

McKeith, I. G. et al. (2005). Diagnosis and management of dementia with Lewy bodies: third report of the DLB Consortium. Neurology, 65, 1863-1872.

McKhann, G., Drachman, D., Folstein, M., Katzman, R., Price, D. and Stadlan, E. M. (1984). Clinical diagnosis of Alzheimer's disease. Report of the NINCDS/ADRDA Work Group, under the auspices of the Department of HHS Task Force on Alzheimer's disease. Neurology, 34, 939.

Mioshi, E., Dawson, K., Mitchell, J., Arnold, R. and Hodges, J. R. (2006). The Addenbrooke's Cognitive Examination Revised (ACE-R): a brief cognitive test battery for dementia screening. International fournal of Geriatric Psychiatry, 21, 1078-1085.

Neary, D., Snowden, J. S., Northen, B. and Goulding, P. (1988). Dementia of frontal lobe type. Fournal of Neurology, Neurosurgery and Psychiatry, 51, 353-361.

Roman, G. C. et al. (1993). Vascular dementia: diagnostic criteria for research studies. Report of the NINDS-AIREN International Workshop. Neurology, 43, 250-260.

Petersen, R. C., Smith, G. E., Waring, S. C., Ivnik, R. J., Kokmen, E. and Tangelos, E. G. (1997). Aging, memory, and mild cognitive impairment. International Psychogeriatrics, 9, 65-69.

ViCtor Michael Aziz,
PADMAVATHY SRINIVASAlU Gopinath ${ }^{2}$
AND GEORGIA BAILY ${ }^{2}$
${ }^{1}$ Cwm Taf Health Board, Mental Health Unit, Cynon
Valley Hospital, New Road, Mountain Ash CF45 4BZ,
UK
${ }^{2}$ Cardiff University School of Medicine, Cardiff, Wales,
UK
Email: victoraziz@doctors.org.uk

Nerve growth factor (NGF), a trophic factor involved in the development, maintenance, and 